\title{
A STUDY ON THE EFFECT OF DIETARY FACTORS ON FUNCTIONAL GASTROINTESTINAL DISORDERS IN WOMEN OF HA'IL REGION IN SAUDI ARABIA
}

\author{
RAMADEVI KUNDUR ${ }^{1}$, KALYAN VISWANATH REDDY LINGALA ${ }^{2}$, ABEER RAWAF MOAYD ALRSHEDI ${ }^{1}$
}

${ }^{1}$ Department of Clinical Laboratory Science, College of Applied Medical Sciences, University of Ha'il, Kingdom of Saudi Arabia. ${ }^{2}$ Department of Health Services Administration, College of Public Health and Health Informatics, University of Ha'il, Kingdom of Saudi Arabia. Email: ramadevipatel@gmail.com

Received: 16 March 2018, Revised and Accepted: 25 April 2018

ABSTRACT

Objective: The aim of this study is to assess the prevalence of functional gastrointestinal disorders (FGIDs) among the women population of the Ha'il region in Saudi Arabia. It also includes the analysis of the effect of dietary factors on FGIDs and to evaluate if any significant correlation exists between the type of diet and FGIDs.

Methods: A cross-sectional study was conducted at the Maternity and Children's Hospital and Ha'il General Hospital in the Ha'il Province of Saudi Arabia with 501 random women patients who had visited these hospitals. Data were collected through a questionnaire and analyzed using the statistical program SPSS-21.

Results: In this study, $50 \%$ of the women population in Ha'il had at least one of the FGIDs. Acidity (14.4\%) is the prominent FGID followed by gas $(11.8 \%)$ and abdominal pain (11.4\%). The FGIDs increase with age, irrespective of dietary factors. Gas formation was observed to be significantly correlated with age $(\mathrm{p}<0.05)$, and unhealthy diet was highly correlated to acidity $(\mathrm{p}<0.01)$ and gas formation $(\mathrm{p}<0.01)$.

Conclusion: FGIDs were observed to be considerably lower if a healthy diet was maintained at least up to 40 years of age. High fiber and protein-rich diet along with fresh fruits and vegetables are known to reduce FGID symptoms to a great extent; hence, dietary changes along with physical activity could help reduce the occurrence of FGIDs.

Keywords: Functional gastrointestinal disorders, Gastroesophageal reflux disease, Irritable bowel syndrome, Functional dyspepsia.

(C) 2018 The Authors. Published by Innovare Academic Sciences Pvt Ltd. This is an open access article under the CC BY license (http://creativecommons. org/licenses/by/4. 0/) DOI: http://dx.doi.org/10.22159/ajpcr.2018.v11i8.25980

\section{INTRODUCTION}

Functional gastrointestinal disorders (FGIDs) are a variable combination of recurrent gastrointestinal (GI) symptoms such as acidity, diarrhea, constipation, and abdominal pain, which cannot be explained by structural or biochemical abnormalities. FGIDs lack identifiable biomarkers and therefore are diagnosed based on their symptom picture. The prevalence of FGIDs in the general population in many countries has been increasing. People with FGIDs are often affected not only physically but also psychosocially, having a massive influence on the society [1]. However, there are no structural abnormalities that can be detected.

The "Rome diagnostic criteria" have classified 24 FGIDs into esophageal, gastroduodenal, bowel, biliary, anorectal, and abdominal pain subcategories. Functional heartburn or gastroesophageal reflux disorder (GERD), functional dyspepsia (FD), and irritable bowel syndrome (IBS) are characterized by often overlapping and most commonly reported symptoms such as abdominal discomfort or pain, acidity or heartburn, gas formation, diarrhea, and constipation. Since most people with FGIDs do not seek medical care, population-based surveys are pivotal in assessing the epidemiology of these conditions. However, the prevalence data are difficult to obtain due to the need for complex longitudinal population-based studies, the fluctuating nature of the disorders, and classification of the subjects. Very few prevalence studies have been performed, and they have reported strikingly different rates [2].

The risk factors associated with FGIDs include lifestyle, emotional stress, sleep problems, and dietary habits. The dietary habits are moving away from traditional food to fast foods which have detrimental effects on health; it is an obvious factor associated with obesity, type 2 diabetes mellitus, cardiovascular disease, food allergy, and hepatotoxicity [3,4]. Various food items such as lactose, fructose, wheat, and caffeine have been implicated to play a role in the prevalence of FGIDs such as IBS and FD. Several mechanisms for food-induced symptoms have been proposed which include food intolerance, food allergy, bacterial overgrowth, altered colonic flora, and alterations in GI physiology after eating. However, the importance of these mechanisms remains unclear [5,6]. The cause of FGIDs remains essentially unknown, but psychological factors, external stress, and environmental factors have been implicated. Although FGID is generally a non-life-threatening disorder that is not associated with survival, FGID patients have an impaired health-related quality of life [7].

In Saudi Arabia, various kinds of GI problems are seen in $61 \%$ of the population, the most common one being the abdominal pain and diarrhea [8]. The prevalence of FD in the population is $7.9-40 \%$ [7] and that of IBS ranges from $9 \%$ to $40 \%$ [9], GERD is at $28.7 \%$ [10]. The prevalence of GERD among the Saudi population is higher than that in Western countries and East Asia [11]. There have been limited and varying reports of prevalence studies of FGIDs in Saudi Arabia. There is no information available on the dietary habits or prevalence of FGIDs, especially among the women population in the Ha'il region of Saudi Arabia. Hence, this study was undertaken to assess the prevalence of FGIDs, the dietary habits and their effects on several FGIDs, and suggests remedial measures.

\section{METHODS}

A cross-sectional quantitative survey was conducted using a structured questionnaire during the period from November 2017 to January 2018 
to address the objectives. The simple random sampling technique was used to collect the data to a target of 550 respondents. However, at the end of the data collection process, 501 valid questionnaires were considered in the final analysis and the response rate was $91 \%$. The respondents consisted of the women of all ages in the Maternity and Children's Hospital and Ha'il General Hospital in Ha'il city, Kingdom of Saudi Arabia. The purpose of the study was explained to the patients, and the questionnaire was given to them to be filled only with their consent. The data collection form included the basic anthropometric details, the frequency of different types of foods consumed, and problems related to the GI tract such as abdominal pain, acidity, indigestion, bloating due to gas, diarrhea, constipation, infections, or any other problems related to the GI tract in detail. Medical history of patients was examined wherever possible. The questionnaire was carefully designed and developed to address the objectives of the study. The questionnaire consisted of closeended responses such as yes/no. The responses were carefully captured and coded in SPSS 21.0 statistical package software for analysis. All reported $p$ values are two sided and differences were considered statistically significant at $\mathrm{p}<0.05$ and $\mathrm{p}<0.01$.

The type of food consumed is categorized as "fatty food," which includes foods fried in oil or butter, sugary foods with high carbohydrates, and foods with low fiber. Protein-rich foods with low carbohydrate and high fiber, fresh fruits, vegetables, and salads are categorized as "healthy food." Three groups were formed based on the regular diet pattern of a person. If the diet includes more than $70 \%$ of fatty foods every day along with high carbohydrates and less fresh fruits or salads, it is an "unhealthy diet." If a person consumes fatty foods once in a week, but if the everyday diet has low carbohydrate and protein rich foods along with salads, it is categorized as "moderately healthy diet." The third group includes people consuming very less fatty foods and eating low carbohydrate, protein-rich foods along with fresh fruits, and salads regularly are on a "healthy diet." These diet patterns are the routine eating habits of the individuals. Only individuals with frequent recurrence of the FGID symptoms for more than 6 months were considered for the study.

\section{RESULTS}

The results obtained after analysis of 501 female participant data using SPSS statistical program Version 21 are presented in this study. The participant demographic characteristics are presented in Table 1 . The age groups of the respondents ranged from 16 to 60 years, with a mean age of $36 \pm 13$ years, $41.5 \%$ participants were between 26 and 40 years, $33.3 \%$ were $>40$ years, and almost $50 \%$ of the participants belonged to the moderate diet group. Healthy diet group had $27.7 \%$ and unhealthy diet group comprised $23.6 \%$ participants.

The frequency of several FGIDs in the population is shown in Fig. 1. In this study, it has been observed that acidity is more prevalent in the population, followed by gas and abdominal pain, constipation, and diarrhea irrespective of age or diet.

The prevalence of FGIDs in different age groups is shown in Fig. 2. In the age-wise study of FGIDs irrespective of diet, acidity (15.1\%) and abdominal pain $(14.3 \%)$ are higher in the $<25$ years age group followed by diarrhea (9.5\%). In the age group of $26-40$ years group,

Table 1: Demographic characteristics of data

\begin{tabular}{ll}
\hline Variables & Frequency (\%) \\
\hline Age & \\
<25 years & 25.1 \\
26-40 years & 41.5 \\
>40 years & 33.3 \\
Diet & \\
Unhealthy diet & 23.6 \\
Moderate diet & 48.9 \\
Healthy diet & 27.7 \\
\hline
\end{tabular}

$\mathrm{N}=501$ acidity $(13.5 \%)$ and gas $(11.5 \%)$ are prevalent. However, gas $(16.2 \%)$ and acidity (16.2\%) are the most prevalent FGIDs after 40 years of age followed by abdominal pain (15\%). Constipation is almost the same irrespective of age. Diarrhea is higher in younger group and older age group $>40$ years.

The effect of dietary habits on the prevalence of FGIDs irrespective of age has been shown in Fig. 3. Acidity (23.7\%), gas (22\%), and abdominal pain $(14.4 \%)$ are the highest FGIDs among the unhealthy diet group followed by the moderate diet group. Healthy diet group shows comparatively less FGIDs.

The effect of diet on the prevalence of FGIDs in three age groups has been observed. In the unhealthy diet group, acidity (35.7\%), abdominal pain $(28.6 \%)$, and gas $(21.4 \%)$ are the highest FGIDs. In the moderate diet group, where fast foods are consumed occasionally, abdominal pain $(21.6 \%)$, acidity $(16.2 \%)$ is higher, and diarrhea $(13.5 \%)$ is increased. The occurrence of FGIDs is considerably less in the younger age group (Fig. 4).

The FGIDs in the middle age group of 26-40 years are shown in Fig 5. FGIDs are lower in healthy diet group. The moderate healthy group has increased FGIDs such as acidity (13\%), gas (10\%), constipation (10\%), and abdominal pain (9\%). The unhealthy eaters have the highest levels FGIDs, acidity (20\%) and gas (18.3\%) followed by constipation (11.7\%).

In the age group $>40$ years, healthy diet group has elevated levels FGIDs such as acidity (25\%), gas (12.5\%), and abdominal pain (12.5\%). Moderate diet does not show very high elevations, but unhealthy diet showed a prominent increase in acidity (26.1\%), gas (26.1\%), and abdominal pain (26.1\%) are the highest (Fig. 6).

The correlation coefficients among the variables such as age, healthy, moderately healthy, unhealthy diet, and FGIDs are shown in Table 2. The results reveal that there is a significant correlation existing between age, type of diet, and FGIDs. There is a strong positive correlation between age and gas formation ( 0.010 at $\alpha=0.05$ level), unhealthy diet and gas formation ( 0.000 at $\alpha=0.01$ level), and unhealthy diet and acidity ( 0.001 at $\alpha=0.01$ level).

\section{DISCUSSION}

Acidity, abdominal pain, gas formation, diarrhea, and constipation are common overlapping symptoms of major GI-related disorders such as GERD, IBS, FD, and peptic ulcers. GERD is mainly due to the reflux of gastric contents into the esophagus with epigastric pain and heartburn as common symptoms. Often, FD is caused by a peptic ulcer or acid reflux. Diarrhea and constipation are more prominent in IBS. In recent years, the number of people suffering with FGIDs has increased. These symptoms implicate not only the existence of FGIDs but also malabsorption syndrome, food allergies, and food intolerance [12]. There are varying reports regarding the prevalence of FGIDs in KSA ranging from 9 to $40 \%$. These diverse prevalence rates within the different parts of the country suggest multifactorial etiological elements [10].

In this study, $50 \%$ of the women have at least one of the FGIDs among which, acidity, gas formation, and abdominal pain were highest in women population of Ha'il region irrespective of age or diet. In another similar study about the pattern of GI diseases in the eastern region of Saudi Arabia, it was found that $75 \%$ of people suffered from abdominal pain followed by diarrhea [8]. In another KSA study, $45 \%$ of population was observed to be suffering from acid reflux symptoms [13]. In the present study, it has been observed that $14 \%$ of the women population in Ha'il region suffers from acidity problem which is a major symptom of GERD. GERD is more prevalent in KSA than in western countries and East Asia, affecting an estimated $20-30 \%$ of the population, and it is the most common outpatient diagnosis in gastroenterology and has a high prevalence in the general population. The latest report of GERD prevalence is at $28.7 \%$ in KSA [11]. As many as $40 \%$ of patients with 


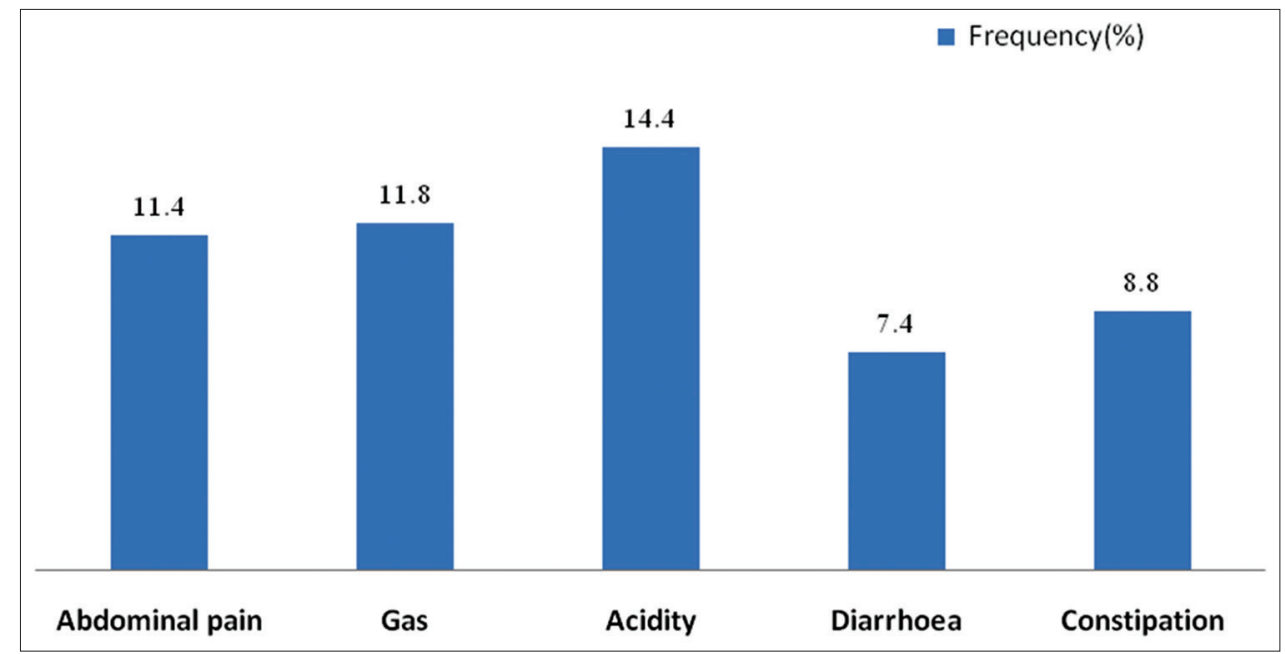

Fig. 1: Frequency of several functional gastrointestinal disorders in the population. $\mathrm{N}=501$

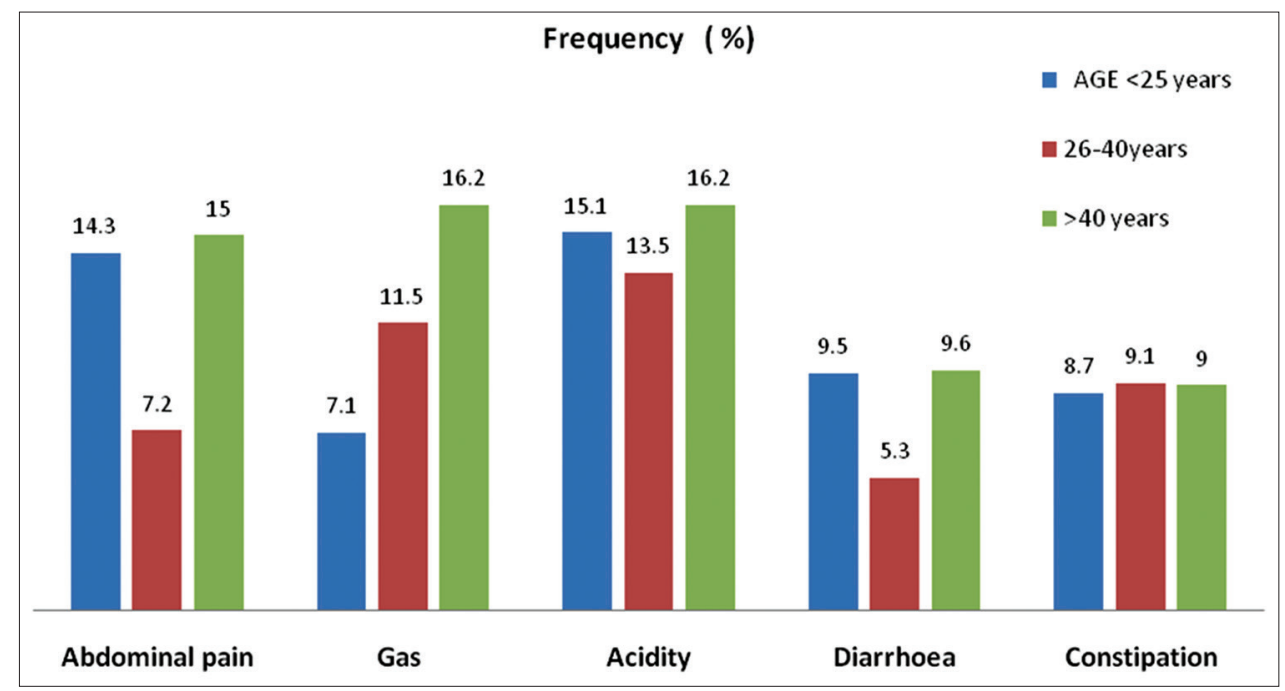

Fig. 2: Frequency of functional gastrointestinal disorders in different age groups

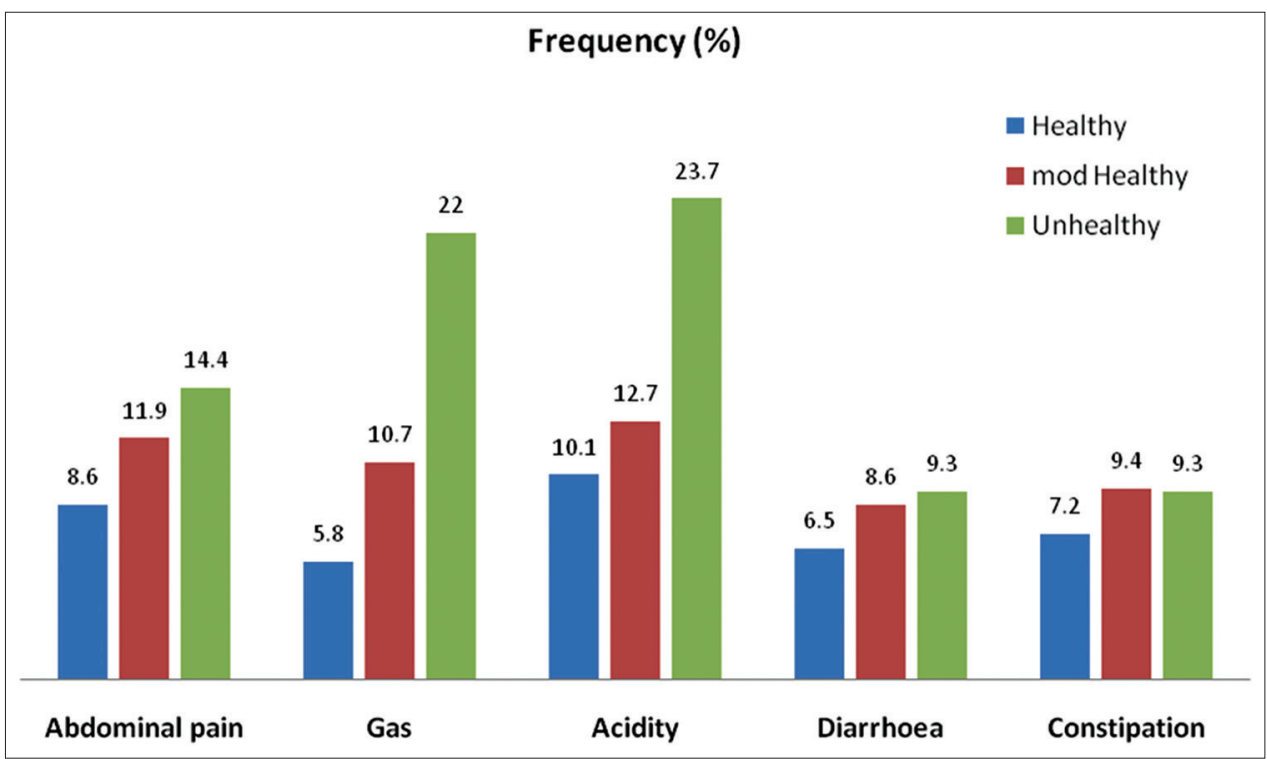

Fig. 3: Frequency of several functional gastrointestinal disorders with different dietary habits. Mod healthy: Moderately healthy 
Table 2: Correlation between age, diet, and FGIDs

\begin{tabular}{|c|c|c|c|c|c|}
\hline Variables & Pain & Gas & Acidity & Diarrhea & Constipation \\
\hline \multicolumn{6}{|l|}{ Age } \\
\hline Pearson correlation & 0.011 & 0.115 & 0.031 & 0.030 & -0.006 \\
\hline Sig. (two-tailed) & 0.805 & $0.010^{*}$ & 0.492 & 0.505 & 0.901 \\
\hline $\mathrm{N}$ & 501 & 501 & 501 & 501 & 501 \\
\hline \multicolumn{6}{|l|}{ Healthy diet } \\
\hline Pearson correlation & -0.040 & -0.116 & -0.076 & -0.039 & -0.051 \\
\hline Sig. (two-tailed) & 0.377 & 0.380 & 0.089 & 0.388 & 0.259 \\
\hline \multicolumn{6}{|l|}{ Moderately healthy diet } \\
\hline Pearson correlation & 0.002 & -0.048 & -0.059 & -0.001 & 0.035 \\
\hline Sig. (two-tailed) & 0.972 & 0.286 & 0.185 & 0.974 & 0.434 \\
\hline $\mathrm{N}$ & 501 & 501 & 501 & 501 & 501 \\
\hline \multicolumn{6}{|l|}{ Unhealthy diet } \\
\hline Pearson correlation & 0.053 & 0.177 & 0.148 & 0.041 & 0.011 \\
\hline Sig. (two-tailed) & 0.237 & $0.000^{* *}$ & $0.001^{* *}$ & 0.359 & 0.813 \\
\hline $\mathrm{N}$ & 501 & 501 & 501 & 501 & 501 \\
\hline
\end{tabular}

*Correlation is significant at the 0.05 level (two-tailed). ${ }^{* *}$ Correlation is significant at the 0.01 level (two-tailed).

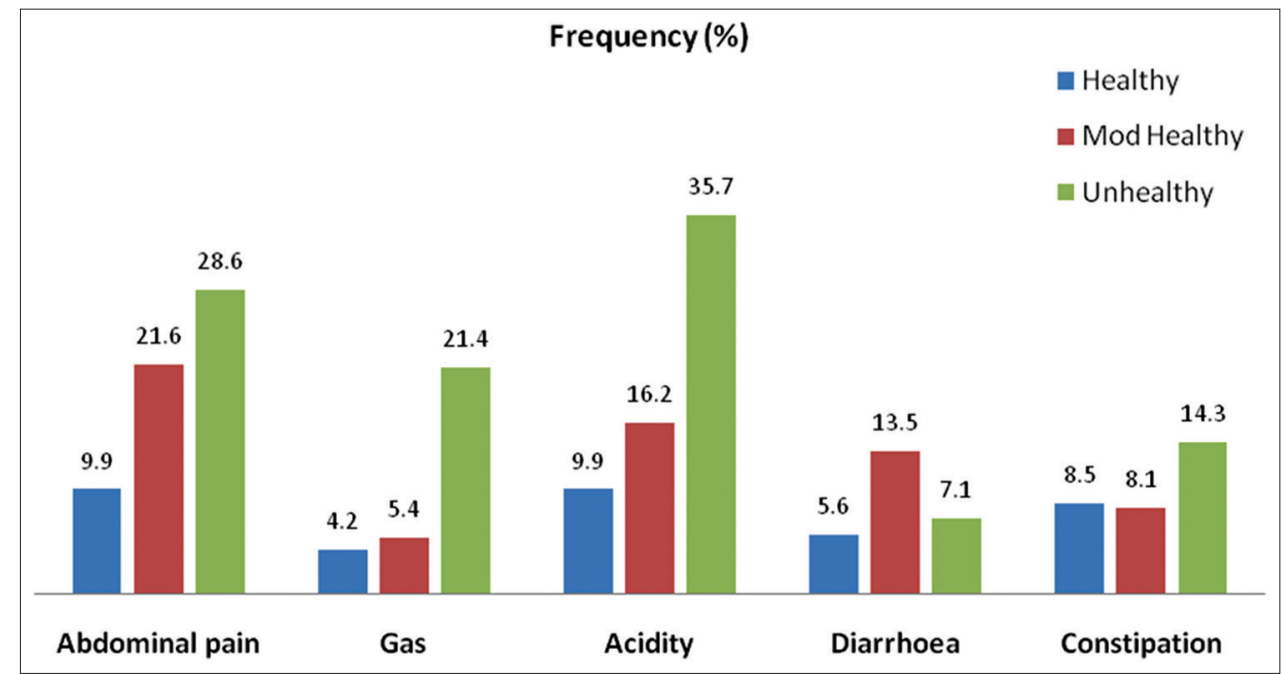

Fig. 4: Frequency of functional gastrointestinal disorders with different dietary habits in the $<25$ years age group. Mod healthy: Moderately healthy

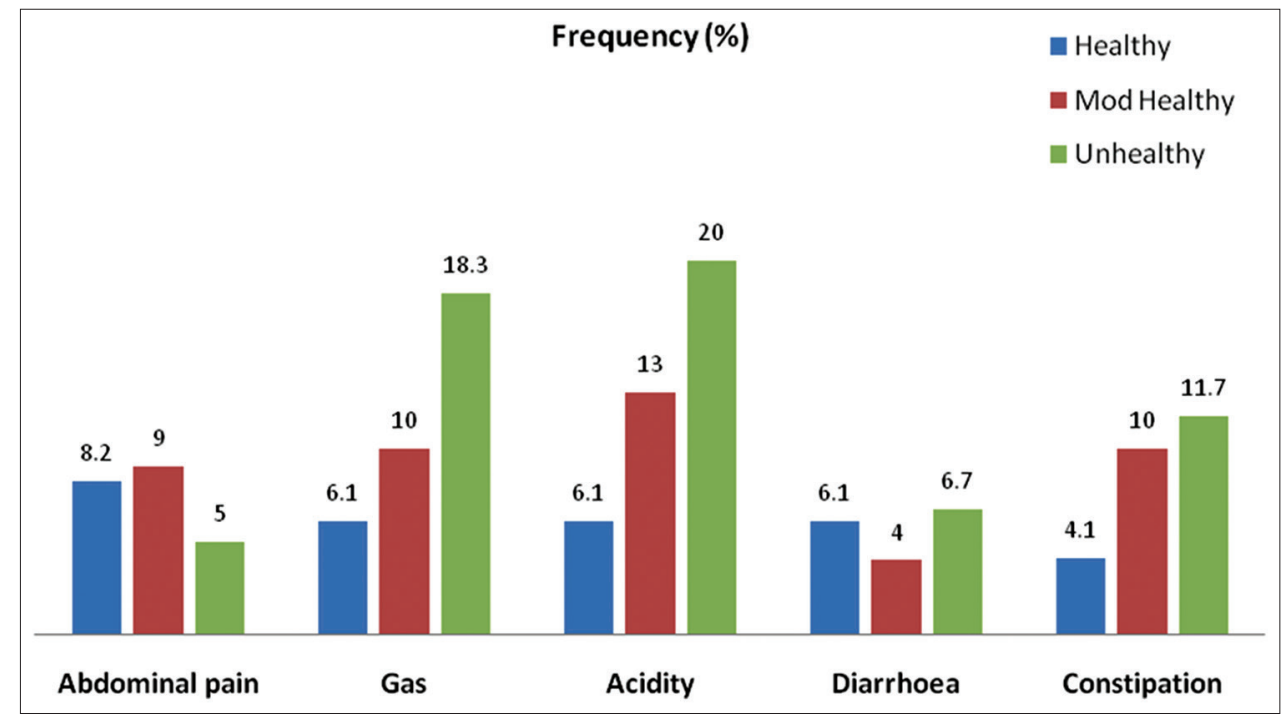

Fig. 5: Frequency of functional gastrointestinal disorders with different dietary habits in 26-40 years age group. Mod healthy: Moderately healthy 


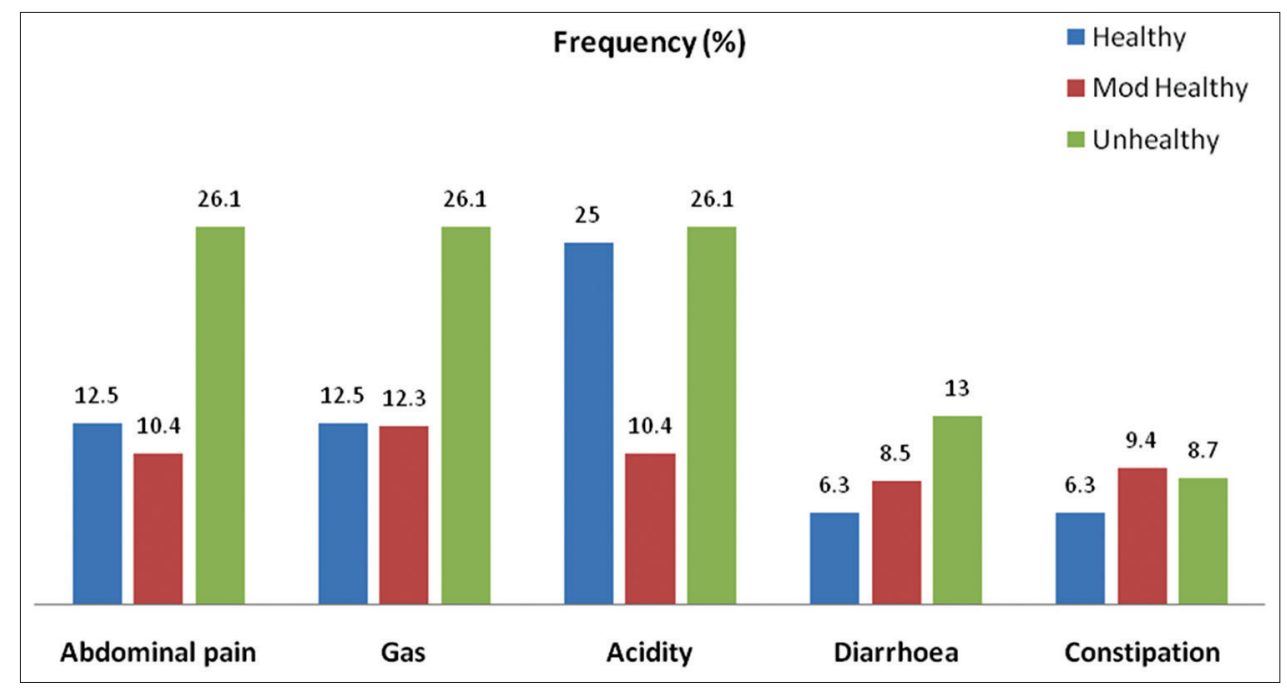

Fig. 6: Frequency of functional gastrointestinal disorders with different dietary habits in the $>40$ years age group. Mod healthy: Moderately healthy

GERD have an inconclusive response to medical therapy. However, now, it is clear that an allergic reaction to food plays an important role, and dietary interventions, as well as biologic agents to block the inflammatory cascade, are being studied [14].

Abdominal pain along with diarrhea or constipation is the most common symptom of IBS. A report from the northern region of KSA showed abdominal pain or discomfort in $37.9 \%$ of patients [15]. The highest reported IBS is $40.7 \%$ in the Qassim region of KSA with no significant gender discrimination [16]. However, in most countries, including Saudi Arabia, almost all of the studies conducted in association with IBS and several other FGIDs such as dyspepsia and GERD have reported a female predominance. In a recent cross-sectional study conducted in Jeddah, KSA, the overall prevalence of IBS was $21 \%$, with a higher prevalence among females at $26 \%$ than among males at $19 \%$ [17]. In an online survey in Japan, the prevalence of both FD and IBS was observed to be higher in women than in men [18]. A similar observation was made in Mexico, where women more frequently reported bowel movements (BMs) associated with the onset of abdominal discomfort and pain and abdominal fullness or bloating than men, constipation too was more common in women than men [19]. Studies found that gender, family history, stress, anxiety, depression, infections, dietary factors, and sleep disorders were associated with FGIDs in KSA $[9,17,18]$.

The role of particular types of foods in the etiology of FGIDs is poorly understood. An attempt has been made in the present study to understand the impact of dietary habits on FGIDs in different age groups. This study definitely confirms age as a factor in the prevalence of FGIDs. Acidity, gas, and abdominal pain were more prevalent as age increased irrespective of dietary factors. Gas formation increased consistently with age and has been the only FGID observed to be significantly correlated with age. The occurrence of FGIDs is observed to be considerably less with a healthy diet, particularly in $<40$ years age groups. Age does appear to have some effect and an increase in FGIDs is seen with advancing years through a healthy diet is maintained. Acidity increased considerably with age even with a healthy diet. Previous studies too reported a significant association of gastroesophageal reflux disease with age $[13,20]$. High-fiber foods such as beans, vegetables, and fruits are associated with a reduction in the prevalence of FGIDs. Fibers are associated with low levels of acid production, and hence, they reduce the acid reflux symptoms $[21,22]$. Likewise, in this study too, it has been observed that the healthy diet group consuming low-fat foods, fruits, and salads with fresh vegetables on a daily basis showed considerably less FGIDs. Acidity, gas, and abdominal pain are observed to be low until the age of 40 if a healthy diet is maintained. An unhealthy diet is observed to be highly correlated to acidity and gas formation in this study.
The unhealthy diet group consuming more of fast foods and fatty foods regularly had more FGIDs than healthy diet group clearly indicating the role of dietary factors. Similar observations have been reported about the role of fried foods, beverages, and spicy foods in causing several FGIDs [21-24]. High-fat intake increases the perception of reflux symptoms and a very low carbohydrate diet protects against reflux [23]. Consumption of spicy foods is directly associated with IBS, particularly in women $[25,26]$. Adherence to fast food patterns contributes to the risk of developing reflux [27]. In the moderate diet group, FGIDs such as abdominal pain, acidity, and diarrhea are higher in the younger group $<25$ years of age. In this group, fast foods and high-fat foods are consumed occasionally; hence, gut sensitivity to food changes or irritation may be more in younger age than in the middle or older age group. In a previous study too, a lower incidence of FD was shown in female patients over 70 years and a higher incidence of IBS in male patients under 29 years [28]. Food intolerance, food allergies, or malabsorption could be the cause of this sensitivity. Food intolerance is an adverse reaction to food which does not involve the immune system. It often causes abdominal cramps or diarrhea due to the foods containing components capable of triggering the reaction [12]. Approximately $50 \%$ of people with IBS report that food worsens their symptoms, drawing attention to the role of food in this context. Evidence encourages researchers to further investigate the role of food intolerance as a major contributor to IBS symptoms $[12,29]$. The food components that induce these functional gut symptoms have been identified. High gluten, fructose, lactose, natural, and added food chemicals such as salicylates, amines, and glutamates, fermentable oligo-, di-, and monosaccharides and polyols (FODMAPs) are known to trigger FGIDs. These are poorly absorbed and exert osmotic effects in the intestinal lumen increasing its water volume and are rapidly fermented by bacteria with consequent bloating and gas production [29,30].

Changes in lifestyle which have been considered in treating FGIDs include small meals, increasing the consumption of fiber, and reducing fatty food, dairy products, carbohydrates, caffeine, alcohol, and food with a high-protein content. Studies are required to assess the efficacy of a dietary approach characterized by low food chemicals. A realistic and efficacious therapeutic approach for FGID symptoms would be that of utilizing food choices through low FODMAPs and other potential dietary strategies [29].

\section{CONCLUSION}

In this study, about $50 \%$ of the women population in Ha'il had at least one of the FGIDs. Acidity is the prevailing FGID followed by gas and abdominal pain. The FGIDs increase with age, irrespective of dietary factors. Gas formation was observed to be significantly correlated 
with age, and unhealthy diet was highly correlated to acidity and gas formation. FGIDs were observed to be considerably lower if a healthy diet was maintained at least up to 40 years of age. High fiber and protein-rich diet along with fresh fruits and vegetables are known to reduce FGID symptoms to a great extent; hence, dietary changes along with physical activity could help reduce the occurrence of FGIDs.

\section{ACKNOWLEDGMENT}

We would like to thank the authorities of Maternity and Children's Hospital and Ha'il General Hospital for their permission and cooperation in conducting this study.

\section{AUTHOR'S CONTRIBUTION}

Conception and design of the study, data interpretation, manuscript and questionnaire preparation, and critical revision of the manuscript are done by Dr. Ramadevi, statistical analysis using SPSS, data analysis, and interpretation and critical evaluation is done by Dr. Kalyan Reddy, and data collection was done by Ms. Abeer.

\section{CONFLICTS OF INTEREST}

The authors declared that they have no conflicts of interest.

\section{REFERENCES}

1. Talley NJ. Functional gastrointestinal disorders as a public health problem. Neurogastroenterol Motil 2008;20:121-9.

2. Corazziari E. Definition and epidemiology of functional gastrointestinal disorders. Best Pract Res Clin Gastroenterol 2004;18:613-31.

3. Ramadevi K, Moody RG. Obesity prevalence and its associated risk factors in women of Ha'il region in Saudi Arabia. J Med Sci Clin Res 2017;5:18504-9.

4. Kesh S, Sarkar D, Manna K. High-fat diet-induced oxidative stress and its impact on metabolic syndrome: A review. Asian J Pharm Clin Res 2016;9:47-52

5. Arafa AA, Ibrahim ES, Fouad EA, Gaber ES. Antibiotic resistance of staphylococci concerning strains included in food industry in Egypt. Int J Pharm Clin Res 2016;8:1583-9.

6. Gomara RE, Halata MS, Newman LJ, Bostwick HE, Berezin SH, Cukaj L, et al. Fructose intolerance in children presenting with abdominal pain. J Pediatr Gastroenterol Nutr 2008;47:303-8.

7. Wahass S, Khalil MS, Al Qurain AA, Yasawy MI. The impact of functional dyspepsia on health-related quality of life in Saudi patients. Saudi J Gastroenterol 2006;12:123-9.

8. Al Quorain AA, Satti MB, Al Gindan YM, Al-Hamdan A. The pattern of lower gastrointestinal disease in the eastern region of Saudi Arabia: A retrospective analysis of 1590 consecutive. Saudi J Gastroenterol 2000;6:27-32.

9. Al Harbi SH. Epidemiology of irritable bowel syndrome in Saudi Arabia. Am J Med Med Sci 2018;8:7-13.

10. Al Karawi MA, Ahmed AM, Shariq S, Mohamed AE. Evaluation of gastro-oesophageal reflux disease results in 40 patients. Saudi Med J 1992; 13:407-11.

11. Alsuwat OB, Alzahrani AA, Alzhrani MA, Alkhathami AM, Mahfouz ME. Prevalence of gastroesophageal reflux disease in Saudi Arabia. J Clin Med Res 2018;10:221-5.
12. Pasqui F, Poli C, Colecchia A, Marasco G, Festi D. Adverse food reaction and functional gastrointestinal disorders: Role of the dietetic approach. J Gastrointestin Liver Dis 2015;24:319-27.

13. Almadi MA, Almousa MA, Althwainy AF, Altamimi AM, Alamoudi HO, Alshamrani HS, et al. Prevalence of symptoms of gastroesopahgeal reflux in a cohort of Saudi Arabians: A study of 1265 subjects. Saudi J Gastroenterol 2014;20:248-54.

14. Massimiliano DP, Rebecca CF. Research advances in esophageal diseases: Bench to bedside. F1000Prime Rep 2013;5:44.

15. Alhazmi AH. Irritable bowel syndrome in secondary school male students in Al Jouf Province, north of Saudi Arabia. J Pak Med Assoc 2011;61:1111-5.

16. Al Khalifah MI, Al Aql AM, Al Mutairi MS, Al Nuqaydan SA, Al Wehaibi AS, Al Jurayyed AM, et al. Prevalence of irritable bowel syndrome among Qassim school teachers, and its impact on their performance and life duties. Saudi Med J 2016;37:817.

17. Al Aqeel MK, Al Owaimer NA, Al Onezan AF, Al Megbel NY, Al Aujan FY. Prevalence of irritable bowel syndrome and its association with anxiety among medical students at King Saud bin Abdulaziz university for health sciences in Riyadh. Pak J Med Sci 2017;33:33-6.

18. Miwa $H$. Lifestyle in persons with functional gastrointestinal disorderslarge-scale internet survey of lifestyle in Japan. Neurogastroenterol Motil 2012;24:464-71.

19. Schmulson M, Adeyemo M, Gutiérrez RG, Charúa GL, Farfán LB, Ostrosky SF, et al. Differences in gastrointestinal symptoms according to gender in Rome II positive IBS and dyspepsia in a Latin American population. Am J Gastroenterol 2010;105:925-32.

20. Yönem Ö, Sivri B, Özdemir L, Nadir I, Yüksel S, Uygun Y. Gastroesophageal reflux disease prevalence in the city of Sivas. Turk J Gastroenterol 2013;24:303-10.

21. Ebrahimi MM, Sabour S, Khoshbaten M, Arefhosseini SR, Saghafi AM. Total diet, individual meals, and their association with gastroesophageal reflux disease. Health Promot Perspect 2017;7:155-62.

22. Singh A, Singh S. Dietary fiber content of Indian diets. Asian J Pharm Clin Res 2015;8:58-61.

23. Blaga ST, Negrutiu DE, Palage M, Dumitrascu DL. Food and gastroesophageal reflux disease. Curr Med Chem 2017;15.

24. Alkhathami AM, Alzahrani AA, Alzhrani MA, Alsuwat OB, Mahfouz ME. Risk factors for gastroesophageal reflux disease in Saudi Arabia. Gastroenterol Res 2017;10:294-300.

25. Esmaillzadeh A, Keshteli AH, Hajishafiee M, Feizi A, Feinle BC, Adibi P. Consumption of spicy foods and the prevalence of irritable bowel syndrome. World J Gastroenterol 2013;19:6465-71.

26. Lovell RM, Ford AC. Effect of gender on prevalence of irritable bowel syndrome in the community: Systematic review and meta-analysis. Am J Gastroenterol 2012;107:991-1000.

27. Khodarahmi M, Azadbakht L, Daghaghzadeh H, Feinle BC, Keshteli AH, Afshar $\mathrm{H}$, et al. Evaluation of the relationship between major dietary patterns and uninvestigated reflux among Iranian adults. Nutrition 2016;32:573-83

28. Okumura T, Tanno S, Ohhira M, Tanno S. Prevalence of functional dyspepsia in an outpatient clinic with primary care physicians in Japan. J Gastroenterol 2010;45:187-94.

29. Gibson PR, Shepherd SJ. Food choice as a key management strategy for functional gastrointestinal symptoms. Am J Gastroenterol 2012;107:657-66.

30. Shepherd SJ, Lomer MC, Gibson PR. Short-chain carbohydrates and functional gastrointestinal disorders. Am J Gastroenterol 2013;108:707-17. 